\title{
Competencias directivas y confianza en una empresa privada de servicios
}

Luis MarTín CORTEZ Giraldo ${ }^{1}$

Recibido: 14/05/2020 Aceptado: 08/03/2021 Publicado: 26/07/2021

\begin{abstract}
RESUMEN
El objetivo de esta investigación de tipo no experimental, descriptivo y correlacional fue identificar la relación entre las competencias directivas de los mandos intermedios y la confianza al interior de los equipos de trabajo de una empresa privada de servicios. La muestra estuvo compuesta por el total de la población de la empresa. Se empleó la escala de competencias directivas de García-Lombardia et al. y la dimensión de confianza de la escala de evaluación de equipo de Lencioni; ambas con propiedades psicométricas adecuadas para el estudio. Los resultados evidencian que, con un $99 \%$ de probabilidad, la confianza se relaciona de manera significativa y positiva con las competencias directivas, es decir, a mayor desarrollo de las competencias directivas, mayor confianza al interior de los equipos de trabajo; y del mismo modo, a menor desarrollo de las competencias directivas, menor será el nivel de confianza.
\end{abstract}

Palabras clave: competencias directivas; confianza; equipos de trabajo; competencias estratégicas; competencias intratégicas.

\section{INTRODUCCIÓN}

La eficacia de los equipos de trabajo es, sin duda, un aspecto importante para el buen desempeño de los empleados dentro de una organización, y la confianza es uno de los factores de éxito en el logro de resultados entre quienes forman parte de la empresa.

La confianza se fundamenta en los comportamientos de los directivos y los jefes, lo que favorece la comunicación, el trabajo en equipo, el rendimiento, la satisfacción y el compromiso de los colaboradores, y a su vez hace productiva a la organización (Cardona y Wilkinson, 2009; Chand, 2018).

Lencioni (2003) define la confianza como la convicción de que las intenciones de los miembros del equipo son buenas, por tanto, no existe razón para cuidar lo que se dice sobre los errores, defectos y limitaciones de quienes lo conforman.

Cuando hay confianza en un equipo, los integrantes pueden hablar y ser conscientes de sus falencias y convertirlas en oportunidades de mejora con el fin de potenciar el desarrollo y eficacia del mismo. Así también, cuando existe confianza, aparece la posibilidad de solicitar ayuda y de expresar ideas u opiniones sobre lo que acontece al interior del equipo y de la organización. Es por ello que la confianza es un aspecto fundamental para el desarrollo de la organización; son los líderes quienes promueven las relaciones adecuadas a través de altos niveles de confianza (Yukl, 2008).

Searle y Ball (2004) se refieren a la importancia de la confianza dentro de las organizaciones y resaltan que es un constructo frágil dentro de las empresas, dada la complejidad del proceso de construirla o de recomponerla en el caso de que exista algún evento que ocasione la ruptura de dicho indicador en la relación del trabajador con la organización.

1 Arquitecto por la Universidad Ricardo Palma. Es consultor independiente de Comportamiento Organizacional y Liderazgo. Coach senior(Lima, Perú) ORCID: https://orcid.org/0000-0002-5176-6757.

Autor de correspondencia: luis.cortez.g@hotmail.com 
La confianza organizacional se desarrolla en el momento en que los trabajadores logran orientar su confianza personal hacia la empresa, lo que resulta en la formación de vínculos y familiaridad de acuerdo a las relaciones que se hayan establecido, y según la dependencia e interacción que se desarrolle. Puede ser analizada mediante la confianza que se ponga en el supervisor y la confianza que se genere sobre la institución; la primera alude a la confianza que tiene un subordinado para aceptar las indicaciones frente a las acciones que no logra controlar, mientras que la segunda alude a la confianza en el apoyo y justicia por parte de las autoridades de la empresa (Mollering et al., 2004; Perilla y Gómez, 2017).

Otro factor importante para el desarrollo de toda organización son las competencias directivas. Cardona (1999) las define como las conductas visibles y cotidianas de una persona, que conducen al logro de resultados respecto a su rol directivo, y las clasifica en tres grupos de competencias: estratégicas, intratégicas, y de eficacia personal.

Las competencias estratégicas sirven para generar valor económico; las intratégicas potencian las capacidades de los empleados y fortalecen su compromiso con la organización; y las de eficacia personal desarrollan actitudes y habilidades que permiten a los colaboradores relacionarse de manera efectiva (Cardona, 1999).

La confianza al interior de los equipos de trabajo y las competencias directivas, como elementos importantes en el desarrollo de toda organización, conducen al objetivo de este estudio: determinar la relación que pueda existir entre dichos constructos e identificar las competencias directivas que tienen mayor incidencia en dicha relación.

Las investigaciones sobre las competencias directivas se han ocupado en estudiar cuáles son las más importantes para los directivos de diferentes organizaciones (García-Lombardía, Cardona y Chinchilla, 2001; Tafur y Carrera, 2012), así como las diferencias existentes en el nivel de desarrollo de las competencias directivas en instituciones públicas y privadas (Encomenderos, 2017). Otras investigaciones sobre las competencias directivas han buscado identificar su relación con el clima laboral u organizacional (Angulo, 2016; Sotomayor, 2017), con el nivel de motivación (Figueroa, 2017), y con la autonomía de gestión y liderazgo (Corona et al., 2017).

Una investigación se ha ocupado en estudiar la relación existente entre los comportamientos de traba- jo en equipo de los miembros de la alta dirección y de mandos intermedios, y el trabajo en equipo en los demás niveles de la organización. Raes (2014) buscó identificar las competencias que los equipos directivos necesitan desarrollar para lograr la cohesión y el rendimiento necesarios para influenciar favorablemente la motivación, el funcionamiento y el desempeño de los equipos intermedios y operativos.

Hay investigaciones que, enfocándose en el estudio del trabajo en equipo, consideran la confianza dentro de las dimensiones a evaluar, como es el caso del trabajo de Miranda y Ortega (2016).

Respecto a la variable confianza, la investigación de Cardona y Wilkinson (2009) estudia la manera en que se generan los vínculos de confianza en la relación directivo-colaborador e identifica los comportamientos - del directivo y del colaboradorque contribuyen a dinamizar lo que los autores denominan el "círculo virtuoso de la creación de confianza".

No se encontraron investigaciones que se ocupen a la vez del estudio de las competencias directivas y de la confianza en los equipos de trabajo, de ahí la importancia y el fundamento de esta investigación.

El objetivo principal de esta investigación es identificar la relación entre la confianza y las competencias directivas; además, tiene como objetivos específicos identificar la relación existente entre la confianza y las competencias estratégicas, las competencias intratégicas y las competencias de eficacia personal.

La hipótesis general plantea lo siguiente:

- Existe una relación significativa y positiva entre la confianza y las competencias directivas.

Las hipótesis específicas plantean lo siguiente:

- Existe una relación significativa y positiva entre la confianza y las competencias estratégicas.

- Existe una relación significativa y positiva entre la confianza y las competencias intratégicas.

- Existe una relación significativa y positiva entre la confianza y las competencias de eficacia personal.

La presente investigación es de suma importancia dado que permite conocer la relación existente entre la confianza y las competencias directivas, lo cual ayuda a tener una visión más amplia de estos 
aspectos en el ámbito organizacional y, basándose en ello, generar diversas estrategias que posibiliten mejorar las condiciones de una o ambas variables dentro de una empresa o institución. Con respecto al conocimiento científico, este estudio contribuye en la medida en que puede ser utilizada como referencia para otras investigaciones que aborden las variables estudiadas. Claro está que es necesario replicar el estudio en un ámbito nacional en donde incluya organizaciones públicas y privadas.

Con respecto al alcance, esta investigación se limita a instituciones con características similares; el estudio no puede ser aplicado por igual a toda organización, en vista de que las instituciones públicas tienen una realidad diferente.

\section{Competencias directivas}

Se puede hablar de competencias directivas a partir de los primeros aportes dados por McClelland (1973), quien postula que el éxito dentro de una organización no solo obedece a aspectos como la personalidad o las aptitudes que los sujetos evidencian, sino a un conjunto de competencias o potencialidades que tienen las personas en función de tareas específicas, mediante las cuales se logran los objetivos planteados. Esta dimensión de capacidades permite que el sujeto desempeñe de manera eficiente y eficaz una acción determinada (Pereda et al., 2018).

Según Hellriegel et al. (2010), las competencias gerenciales son un grupo de competencias que tienen que ver con el conocimiento, destreza, actitudes y comportamientos que requiere un ejecutivo para conseguir éxito dentro de una organización. Estos factores, gestionados de buena manera junto a un desarrollo adecuado de la capacidad intelectual, conducirán a una administración efectiva y al rendimiento esperado.

Otros autores hablan de estas competencias en el sentido de habilidades directivas. Riera (2005) señala que las habilidades directivas pueden ser aprendidas por cada una de las personas que lo deseen y tengan la posibilidad de hacerlo, y se rigen basándose en cómo el sujeto logra relacionarse con su entorno y cómo logra realizar de manera eficaz las actividades que le correspondan. Dichas habilidades se centran en aspectos como la comunicación, la motivación, el análisis crítico, el manejo del estrés, la dirección y cómo es que los individuos gestionan su conocimiento para el logro de los objetivos que se plantean dentro de la organización.
Existen diversas clasificaciones de las competencias directivas elaboradas por distintos autores, pero la presente investigación parte del trabajo de Cardona (1999), quien incluye dentro de las competencias directivas a las competencias estratégicas, orientadas a la generación de valor económico; a las competencias intratégicas, que posibilitan el desarrollo profesional de los colaboradores y el fortalecimiento del compromiso y la confianza con la organización; y a las competencias de eficacia personal, que contribuyen favorablemente en las relaciones interpersonales.

Las competencias estratégicas incluyen la visión de negocio, la resolución de problemas, la gestión de recursos, la orientación al cliente, la red de relaciones efectivas y la negociación; las competencias intratégicas incluyen la comunicación, la organización, la empatía, la delegación, el coaching y el trabajo en equipo; y las competencias de eficacia personal la conforman la proactividad, el autogobierno, la gestión personal y el desarrollo personal (Cardona, 1999).

\section{Confianza}

El término "confianza", entendido desde el plano organizacional, tiene una diversidad de definiciones según los estudios que se han realizado bajo diferentes posturas, dentro de las cuales resalta lo que expone Gilbert (1998, citado en Salinas, 2012), quien define la confianza como una creencia que posee una persona dentro de la organización, donde dicha creencia se inclina a que es reconocida y honrada, por lo que el sujeto tiene un compromiso elevado con la organización. Por otro lado, Maguire y Phillips (2008) incluyen el término "expectativa" dentro de la definición de la confianza, entendiéndola como un factor que genera expectativa en el sujeto dentro de la institución y, a través de dicha expectativa, desarrolla conductas previsibles y un sentido benevolente hacia su centro de trabajo, con lo que se obtienen mejores resultados en la organización (Rodríguez, 2018; Olvera et al., 2017).

Otra definición es la que exponen Albrecht y Wackernagel (citados en Santiago, 2001), quienes se refieren a la confianza como la forma en que un sujeto ve al resto de sujetos dentro de una organización, la cual es una conducta dependiente y consistente para la estructura de los juicios y el modo de actuar de dicha persona. Según Lara-Rosano (2011), la confianza consiste en un conjunto de mecanismos, tanto formales como informales, que se generan a través de la interacción entre las personas de una organización por medio de diversos medios jerárquicos, e influencia los procesos estratégicos de la misma. La confianza posee características que provocan en las 
personas valores y generan nuevas formas de relacionarse, trabajar y organizarse, lo que define en gran medida el comportamiento organizacional en una empresa.

A partir de lo expuesto, se puede considerar que la confianza es de suma importancia en toda organización, puesto que implica una serie de procesos que determinan el éxito dentro de la misma. Además, es posible aseverar que la confianza es un capital social vital para toda organización al tratarse del producto de un proceso continuo y dinámico de las relaciones interpersonales dentro de toda institución, que se desarrolla cuando los sujetos logran generalizar su confianza personal a la propia organización (Mollering et al., 2004). Estos vínculos generarán un ambiente grato durante el desarrollo de actividades, por lo que todo proceso que se realice puede contribuir a que un sujeto adquiera un nivel de confianza adecuado; así, una persona dentro de una empresa puede desenvolverse de mejor manera potencializando sus fortalezas y propiciando condiciones en donde se logren los objetivos de la misma de mejor manera.

Por otro lado, Castaingts (2008) menciona que cada uno de los trabajadores dentro de la organización requiere de una guía que oriente sus sentimientos y emociones al momento de efectuar cualquier acción; estas personas generalmente se guían a través de creencias, que dependen de la visión del sujeto respecto a cómo se relaciona y cómo comprende el mundo a través de su propia perspectiva.

Otro aspecto importante dentro del tema de la confianza es aquel que la determina como la fuente primordial de cooperación; sin embargo, existe también la posibilidad de que no se dé de la forma más idónea ya que una persona no necesariamente recibe lo que brinda a otros; además, otorgar la confianza a una persona no necesariamente significa que esta va a responder igual o de buena manera. Esta situación es muy frecuente y está ligada a la personalidad de cada individuo (Acedo y Gomila, 2013). Con respecto a lo mencionado, Alter (2002) señala la existencia de un comportamiento egoísta entre colegas, el cual se debe abordar de presentarse, dado que las relaciones de confianza facilitan la cooperación, y esta es la que mejores beneficios puede traer durante el desempeño de los trabajadores dentro de una organización. Ocuparse de esto constituye un proceso complejo en el cual intervienen una serie de variables e incluso la propia personalidad de cada integrante de la organización.

\section{METODOLOGÍA}

La presente es una investigación empírica en la que se logra identificar una cantidad medible a través de pruebas estadísticas tradicionales, lo que permite que sea del tipo cuantitativa (Alfaro, 2012). También es una investigación descriptiva, ya que reseña acontecimientos, contextos y fenómenos, precisando y describiendo hechos y características de aquello que sucede (Hernández et al., 2014). Así mismo, la investigación es de tipo correlacional, pues busca identificar si existe o no relación entre la confianza existente en los equipos de trabajo y las competencias directivas de los jefes inmediatos de los colaboradores evaluados. La investigación cuenta con un diseño no experimental, deja de lado la manipulación de las variables en investigación, lo que significa que no se busca analizar la influencia de una variable sobre otra usando métodos intencionales (Hernández et al., 2014).

La población estuvo conformada por todos los empleados de una empresa privada de servicios que se encuentra en la ciudad de Lima, la cual asciende a un total de 40 colaboradores entre varones y mujeres, cuyas edades superan los 25 años. La técnica de muestreo empleada para el presente estudio fue la de tipo censal. Del total de la muestra, 2 personas no asistieron el día de la aplicación de los instrumentos y 8 no dieron su consentimiento, por lo que la escala de medición de competencias directivas y la escala de confianza en los equipos de trabajo se aplicó a un total de 30 colaboradores.

El desarrollo de este estudio se planificó de manera sistemática y organizada, lo que condujo al logro de los objetivos de la investigación. Una de las primeras acciones realizadas fue reunirse con los directivos de la organización para coordinar la recolección de los datos. Luego, una vez realizadas las coordinaciones, se programó la aplicación de las escalas de medición. Los instrumentos utilizados para el desarrollo del estudio fueron el "Cuestionario de competencias directivas" de García-Lombardía et al. (2001) y la "Escala de evaluación del equipo" (Team Assessment) de Lencioni (2003), de la que se tomó solo los ítems correspondientes a la dimensión de la confianza. La primera con confiabilidad de 0.76 (alfa de Cronbach) y validez de 0.76 (Kaiser Olkin) con varianza de $55.11 \%$, y la segunda con confiabilidad de 0.95 y validez de 0.93 con varianza de $59.33 \%$. Los instrumentos utilizados fueron elegidos en vista de que se ajustaban mejor a la población en que se llevó a cabo el estudio y porque se carece de instrumentos que midan los componentes planteados en esta investigación, por 
lo que sería importante realizar más estudios sobre instrumentos que se basen en la teoría propuesta o que se adapten mejor los instrumentos existentes a la realidad actual.

Seguidamente, se procedió a explicar los objetivos del estudio a los participantes y la forma de responder a los instrumentos. Se informó que la participación era voluntaria y que los cuestionarios eran anónimos. Una vez recolectada la información, se procedió a armar la base de datos correspondiente, teniendo en cuenta los valores necesarios, y una vez establecida y depurada, se procedió con el análisis estadístico. Primero, se analizaron los niveles de cada una de las variables estudiadas a través de las medidas de tendencia central; luego, se evaluó la distribución de la muestra con el Kolmogorov-Smirnov (K-S), que definió la bondad de ajuste entre dos distribuciones de probabilidad entre sí, lo que permitió identificar los estadísticos más idóneos para alcanzar los objetivos propuestos; y, finalmente, se utilizó el índice de correlación de Spearman para el cálculo de las correlaciones, con el cual se determinó la relación entre las variables estudiadas.

\section{RESULTADOS}

A partir de los datos demográficos recogidos entre los colaboradores evaluados $(n=30)$, se puede afirmar que el $35.7 \%(n=11)$ son varones, mientras que el $63.3 \%(n=19)$ son mujeres, tal y como podemos apreciar en la Tabla 1.

Tabla 1. Frecuencia de los colaboradores evaluados según el sexo.

\begin{tabular}{|lcc|}
\hline & Frecuencia & Porcentaje \\
\hline Varones & 11 & 36.7 \\
Mujeres & 19 & 63.3 \\
\hline Total & 30 & 100.0 \\
\hline
\end{tabular}

Fuente: Elaboración propia.

Respecto a la confianza, se obtuvo una media igual a 11.4 con una desviación estándar de 0.49 ; respecto al total de las competencias directivas se obtuvo una media igual a 94.5 con una desviación estándar de 3.28. De las competencias directivas, las competencias de eficacia personal son las que tuvieron mayor puntuación con una media igual a 34.90 con una desviación de 1.32; a estas les siguieron las competencias estratégicas que alcanzaron una media igual a 30.06 con una desviación estándar de 1.82; y finalmente las competencias intratégicas obtuvieron una media de 29.57 con una desviación de 1.43 (ver Tabla 2). A partir de los resultados obtenidos, se observa que las puntuaciones obtenidas en la presente investigación son de valores altos por parte de cada una de las dimensiones y los totales de las variables estudiadas.

Tabla 2. Estadísticos generales de confianza y las competencias.

\begin{tabular}{|lccc|}
\hline & N & Media & DE \\
\hline Confianza & 30 & 11.4 & 0.49 \\
Competencias estratégicas & 30 & 30.06 & 1.82 \\
Competencias intratégicas & 30 & 29.57 & 1.43 \\
Competencias de eficacia personal & 30 & 34.90 & 1.32 \\
Total de competencias directivas & 30 & 94.53 & 3.28 \\
\hline
\end{tabular}

Fuente: Elaboración propia.

Respecto a la distribución de la muestra, se realizó el análisis de normalidad de la muestra (ver Tabla 3 ), en donde se observó que los valores son significativos con un nivel de confianza del 99\% $(p=0.00$; $p<0.01$ ), por lo que se concluye que la distribución no es normal y es por lo que se realizó el siguiente análisis con estadísticos no paramétricos. Para el análisis de las correlaciones entre la confianza y las competencias directivas se utilizó el coeficiente de correlación de Spearman.

Tabla 3. Distribución de la muestra.

\begin{tabular}{|lccc|}
\hline & \multicolumn{3}{c|}{ Kolmogorov-Smirnov } \\
& Estadístico & gl & Sig. \\
\hline Total de competencias directivas & 0.31 & 30 & 0.00 \\
Confianza & 0.39 & 30 & 0.00 \\
Competencias estratégicas & 0.30 & 30 & 0.00 \\
Competencias intratégicas & 0.19 & 30 & 0.00 \\
Competencias de eficacia personal & 0.30 & 30 & 0.00 \\
\hline
\end{tabular}

Fuente: Elaboración propia.

\section{Contraste de hipótesis}

En la Tabla 4, se puede ver la correlación de Spearman entre la confianza y el puntaje total de las competencias directivas y sus dimensiones respectivas, es decir, las competencias estratégicas, intratégicas y de eficacia personal. Los resultados arrojan valores cuya significancia es equivalente a $p=0.00$ $(p<0.01)$ y alcanzan un nivel del $99 \%$ de probabilidad de que existe una relación significativa entre la confianza y las competencias directivas. Asimismo, por el valor obtenido (rho $=0.72$ ) y por la clasificación realizada por Martínez y Campos (2015) del rango de coeficientes de correlación, la relación es positiva alta, por lo que se puede decir que a 
mayor desarrollo de las competencias directivas, mayor será la confianza al interior de los equipos de trabajo, y lo mismo en el caso inverso, a menor desarrollo de la primera variable, menores o bajos resultados en la segunda. Respecto a las dimensiones de las competencias directivas, los resultados indican que hay un nivel del $95 \%$ de probabilidad ( $p$ $=0.03 ; p<0.05$ ) de que exista una relación significativa entre la confianza y las competencias estratégicas, y que por el valor obtenido (rho $=0.40$ ), la relación es positiva moderada (Martínez y Campos, 2015). Se puede afirmar entonces que la relación entre la confianza y las competencias orientadas a la generación de resultados o valor económico (estratégicas) se asemeja a la relación de la confianza con el total de las competencias directivas; sin embargo, no es tan fuerte su relación como la citada anteriormente.

Tabla 4. Correlación entre la confianza y las competencias directivas.

\begin{tabular}{|llc|}
\hline & \multicolumn{2}{c|}{ Confianza } \\
& rho & p \\
\hline Competencias estratégicas & $0.40^{*}$ & 0.03 \\
Competencias intratégicas & $0.72^{* *}$ & 0.00 \\
Competencias de eficacia personal & $0.59^{* *}$ & 0.00 \\
Total de competencias & $0.72^{* *}$ & 0.00 \\
\hline
\end{tabular}

*La correlación es significativa en el nivel 0.05 (bilateral).

${ }^{* *}$ La correlación es significativa en el nivel 0.01 (bilateral).

Fuente: Elaboración propia.

Por otro lado, hay un nivel del 99\% de probabilidad $(p=0.00 ; p<0.01)$ de que exista una relación significativa entre la confianza y las competencias intratégicas, y la confianza y las competencias de eficacia personal; sin embargo, por los valores rho obtenidos, existe una diferencia en la interpretación de estas relaciones significativas. Respecto a las competencias intratégicas, el valor encontrado es rho $=0.72$, por lo que se puede afirmar que existe una relación positiva alta entre la confianza y estas competencias relacionadas con el saber desarrollar las aptitudes y capacidades de los colaboradores (Martínez y Campos, 2015). Respecto a las competencias de eficacia personal, el valor hallado es rho $=0.59$, por lo que se puede decir que existe una relación positiva moderada entre la confianza y estas competencias que facultan a los mandos intermedios para manejar de manera adecuada las relaciones interpersonales y desarrollar en sus equipos buena disposición e identificación con la organización (Martínez y Campos, 2015).

\section{DISCUSIÓN}

Los resultados de la investigación muestran que existe una relación significativa y positiva entre las competencias directivas y la confianza dentro de los equipos de trabajo, es decir, a mayor desarrollo de las competencias directivas, mayor confianza al interior de los equipos de trabajo; y de manera inversa se puede decir que, a menor desarrollo de las competencias directivas, menor nivel de confianza entre los miembros de los equipos de trabajo.

El nivel de desarrollo de las competencias directivas de quienes conducen los equipos en una organización guarda una relación directa y positiva con el clima laboral, tal como lo demuestran los resultados de los estudios de Angulo (2016) y Sotomayor (2017). Estas investigaciones ponen énfasis en el desarrollo de la comunicación, el trabajo en equipo, el liderazgo, etc. Uno de estos estudios recomienda que los directivos establezcan relaciones de confianza con los demás miembros de la organización para propiciar un mejor clima de trabajo (Angulo 2016). A su vez, el otro estudio recomienda que los directivos evidencien un comportamiento ético y transparente, en vista de que ello propicia un clima de confianza al interior de la organización (Sotomayor, 2017).

El desarrollo de competencias directivas genera cambios y/o mejoras en las disposiciones personales de los miembros de la organización; un ejemplo de ello es el incremento de los niveles de motivación en los colaboradores, tal como lo señalan los resultados del estudio de Figueroa (2017). Otro ejemplo es el desarrollo de competencias de miembros de la alta dirección para trabajar mejor entre pares, como equipo directivo unido; los comportamientos de estas prácticas se transfieren a los mandos intermedios y al resto de la organización, lo que influye en el nivel de confianza, en la mejora de las relaciones interpersonales, en la actitud colaborativa y en el rendimiento conjunto, tal como lo muestra el estudio de Raes (2014).

Los directivos de las organizaciones valoran más algunas de las competencias directivas respecto de otras. Dentro de las competencias que ellos consideran están la comunicación, el trabajo en equipo y el liderazgo, sumadas a la orientación al cliente, la integridad o compromiso ético, y el coaching, entre otras, según los resultados de los estudios de Tafur y Carrera (2012) y de García-Lombardía et al. (2001). Esta última investigación obtuvo como resultado que las empresas desean de sus directivos un nivel de desarrollo similar de los tres tipos de competencias directivas -estratégicas, intratégicas y de eficacia 
personal-, con énfasis en las competencias intratégicas. Este resultado puede complementar, a nivel de conocimiento, el resultado de la presente investigación, que muestra una relación positiva y alta entre las competencias intratégicas y la confianza al interior de los equipos de trabajo.

De igual forma, Corona et al. (2017), en su estudio para la elaboración de un modelo de competencias directivas para la autonomía de gestión y liderazgo en supervisores, muestran que las competencias intratégicas fueron las que contaron con mayor preferencia para el objetivo que tuvo el estudio. Dicha investigación también puede complementar los resultados obtenidos en la presente investigación.

Por otra parte, la confianza junto con el conflicto productivo son las dimensiones del trabajo en equipo que ejercen mayor influencia sobre los procesos de cambio organizacional, tal como lo muestra el estudio de Miranda y Ortega (2016).

Asimismo, el vínculo de confianza entre directivo y colaborador tiene una dinámica cuyos insumos son los comportamientos de quienes dirigen los equipos en las organizaciones; si estos comportamientos generan confianza en el colaborador, este "tiende a corresponder con conductas también positivas", tal como lo indica el estudio de Cardona y Wilkinson (p. 23, 2009). En el comportamiento organizacional es posible predecir el comportamiento de los colaboradores al observar el de los dirigentes.

A partir del análisis de las variables del estudio, puede decirse que, si en un ambiente laboral existe mayor confianza al interior de un equipo de trabajo, es probable que el jefe directo de dicho equipo posea mayor nivel de desarrollo de sus competencias directivas; así, según la presente investigación, se destacan en primer lugar sus competencias intratégicas, seguidas de las estratégicas y de las de eficacia personal. Como dicha relación es directa y positiva, se infiere que, en una organización en la que un directivo no tenga desarrolladas sus competencias directivas, es probable que en el equipo de trabajo que está a su cargo no exista mucha confianza entre los miembros que lo componen. Es necesario precisar que la obtención de mayores niveles en una de las variables no se fundamenta únicamente en el desarrollo de la otra variable; si bien se ha identificado que existe relación entre la confianza y las competencias directivas, no se puede afirmar una causalidad única por parte de alguna de ellas.

Adicionalmente, puede decirse que, si una organización necesita mejorar el desempeño de los trabajadores, podría dedicarse a desarrollar las competencias directivas de quienes tienen personas a cargo y así elevar el nivel de confianza dentro de sus equipos, de modo que contribuya a mejorar el rendimiento de los colaboradores y así estar en mejores condiciones de alcanzar los objetivos trazados.

\section{CONCLUSIONES}

1. Existe una relación significativa y positiva entre la confianza y las competencias directivas, por lo que se puede determinar que, a mayores niveles de las competencias directivas, mayores serán los niveles de confianza entre los colaboradores.

2. Existe una relación significativa y positiva entre la confianza y las competencias estratégicas, por lo que se puede determinar que, en tanto mayores sean los niveles de las competencias estratégicas, mayores serán los niveles de confianza dentro de los equipos de trabajo.

3. Existe una relación significativa y positiva entre la confianza y las competencias intratégicas, por lo que se puede determinar que, en tanto mayores sean los niveles de las competencias intratégicas, mayores serán los niveles de confianza al interior de los equipos de trabajo.

4. Existe una relación significativa y positiva entre la confianza y las competencias de eficacia personal, por lo que se puede determinar que, en tanto mayores sean los niveles de las competencias de eficacia personal, mayores serán los niveles de confianza entre los miembros de los equipos de trabajo.

5. Los resultados se pueden generalizar en organizaciones cuyas características se asemejen a la empresa donde se desarrolló el presente estudio.

6. Estos resultados tienen relevancia en el hecho de que si se desea incrementar la confianza de los trabajadores, es necesario reforzar las competencias directivas de sus líderes, lo cual conllevará una serie de beneficios en el desarrollo de la organización.

\section{REFERENCIAS BIBLIOGRÁFICAS}

[1] Acedo, C., y Gomila, A. (2013). Confianza y cooperación. Una perspectiva evolutiva. Contrastes, 18, 221-238. 
[2] Alfaro, C. (2012). Metodología de investigación científica aplicado a la ingeniería [Informe final de proyecto de investigación]. Instituto de Investigación de la Facultad de Ingenierías Eléctrica y Electrónica de la Universidad Nacional del Callao. Recuperado de https:// unac.edu.pe/documentos/organizacion/vri/ cdcitra/Informes_Finales_Investigacion/IF_ ABRIL_2012/IF_ALFARO\%20RODRIGUEZ_FIEE.pdf

[3] Alter, N. (2002). Théorie du don et sociologie du monde du travail. Revue du MAUSS, 2(20), 263-285. Recuperado de https://doi. org/10.3917/rdm.020.0263

[4] Angulo, D. (2016). Determinación de las competencias directivas y del clima laboral existente en el I.E. "Los Positos", Mórrope, Lambayeque. (Tesis de pregrado). Universidad Católica Santo Toribio de Mogrovejo, Chiclayo. Recuperado de http://tesis.usat.edu.pe/ handle/20.500.12423/164

[5] Cáceres, L., y Gallegos, W. (2018). Importancia de las competencias directivas desde la percepción de los empresarios arequipeños. Contabilidad y Negocios 13(26), 109-128. Recuperado de https://doi.org/10.18800/ contabilidad.201802.007

[6] Cardona, P. (diciembre, 1999). En busca de las competencias directivas. IESE Revista de antiguos alumnos, 19-26.

[7] Cardona, P., y Wilkinson, H. (2009). Cómo crear el círculo virtuoso de la confianza. IESE Insight, (3), cuarto trimestre 20-27.

[8] Castaingts, J. (2008). Antropología simbólica y neurociencia. Alteridades, 18(35), 129-138.

[9] Chand, S. R. (2018). Inspira: Cómo crear una cultura organizacional poderosa. Pensilvania, Estados Unidos de América: Whitaker House.

[10] Corona, L., Ramírez, G., y Vázquez, E. (2017). Modelo de competencias directivas para la autonomía de gestión y liderazgo en supervisores de educación básica en el marco de las reformas educativas en México. Killkana Sociales, 1(3), 23-28. Recuperado de https:// doi.org/10.26871/killkana_social.v1i3.59

[11] Encomenderos, Y. (2017). Competencias directivas en instituciones educativas públicas y privadas del distrito de Tarapoto, 2017. (Tesis de maestría). Universidad César Vallejo, Lima. Recuperado de http://repositorio.ucv.edu.pe/ handle/UCV/12785
[12] Figueroa, J. (2017). Competencias directivas y nivel de motivación en docentes de secundaria de instituciones educativas privadas, Bellavista - Callao, 2015. (Tesis de maestría). Universidad César Vallejo, Lima. Recuperado de https://repositorio.ucv.edu.pe/ handle/20.500.12692/8344

[13] García-Lombardía, P., Cardona, P., y Chinchilla, M. (2001). Las competencias directivas más valoradas. Recuperado de https://media.iese. edu/research/pdfs/OP-01-04.pdf

[14] Hellriegel, D., Jackson E. y Slocum J. (2010). Administración: Un enfoque basado en competencias. Ciudad de México, México: Cengage Learning Editores.

[15] Hernández, R., Fernández, C., y Baptista, P. (2014). Metodología de la Investigación (6 ed.). México D.F., México: McGraw-Hill.

[16] Lara-Rosano, F. (2011). Organizaciones Complejas. En J. Flores y G. Martinez (Compiladores), Encuentros con la complejidad. Ciudad de México, México: Siglo XXI Editores.

[17] Lencioni, P. (2003). Las cinco disfunciones de un equipo. Barcelona, España: Ediciones Urano.

[18] Maguire, S., y Phillips, N. (2008). Citibankers at Citigroup: a study of the loss of institutional trust after a merger. Journal of Management Studies, 45(2), 372-401. Recuperado de https:// doi.org/10.1111/j.1467-6486.2007.00760.x

[19] Martínez, A. y Campos, W. (2015). Correlación entre actividades de interacción social registradas con nuevas tecnologías y el grado de aislamiento social en los adultos mayores. Revista mexicana de Ingeniería Biomédica, 36(3), 181-190.

[20] McClelland, D. (1973). Testing for Competence rather than for "Intelligence". American Psychologist, 28(1), 1-14.

[21] Miranda, A., y Ortega, M. (2016). Evaluación de las características de trabajo en equipo en las empresas del sector educativo particular de la ciudad de Cajamarca-2016. (Tesis de pregrado). Universidad Privada Antonio Guillermo Urrelo, Cajamarca. Recuperado de http://repositorio.upagu.edu.pe/bitstream/ handle/UPAGU/258/IT\%20Alexis $\% 20 y \% 20$ Miguel. pdf?sequence $=1$ \&isAllowed $=y$

[22] Mollering, G., Bachmann, R., y Lee, S. H. (2004). Understanding organizational trust - foundations, constellations, and issues of 
operationalisation. Journal of Managerial Psychology, 19(6), 555-570. Recuperado de http://dx.doi.org/10.1108/02683940410551480

[23] Olvera, J., LLorens, S., Acosta, H., y Salanova, M. (2017). El liderazgo transformacional y la confianza como antecedentes del desempeño en equipo en el ámbito sanitario. Anales de Psicología/Annals of Psychology, 33(2), 365375.

[24] Pereda, S., Berrocal, F., y López, M. (2002). Gestión de recursos humanos por competencias y gestión del conocimiento, Dirección y Organización, (28), 43-54.

[25] Perilla, L., y Gómez, V. (2017). Relación del estilo de liderazgo transformacional con la salud y el bienestar del empleado: el rol mediador de la confianza en el líder. Revista de Psicología del Trabajo y de las Organizaciones, 33(2), 95108.

[26] Raes, A. (2014). Predicar con el ejemplo. E trabajo en equipo empieza en la alta dirección. Revista IESE Insight, (20), 31-38.

[27] Riera, J. (2005). Habilidades en el deporte. Barcelona, España: INDE Publicaciones.

[28] Rodríguez Egea, N. (2018). Liderazgo auténtico, clave de Confianza. (Trabajo final de máster). Universitat Jaume I, Castelló de la Plana. Recuperado de http://repositori.uji. es/xmlui/bitstream/handle/10234/180476/ TFM_2018_RodriguezEgea_Natalia. pdf?sequence $=1 \&$ isAllowed $=y$
[29] Salinas, M. (2012). Elementos que influyen en el establecimiento de la confianza dentro de las organizaciones. (Tesis de doctorado). Universidad Autónoma de Nuevo León, San Nicolás de Garza.

[30] Santiago, A. (2001). Desarrollo y validación de la escala de confianza organizacional. (Tesis de doctorado). Pontificia Universidad Católica de Puerto Rico, Ponce.

[31] Searle, R., y Ball, K. (2004). The Development of Trust and Distrust in a Merger. Journal of Managerial Psychology, 19(7), pp. 708-721. Recuperado de https://doi. org/10.1108/02683940410559392

[32] Sotomayor, L. (2017). Competencias directivas y clima organizacional en instituciones educativas del distrito El Agustino, 2015 (Tesis de maestría). Universidad César Vallejo, Lima. Recuperado de http://repositorio.ucv.edu.pe/ handle/UCV/8323

[33] Tafur, R., y Carrera, P. (2013). Competencias Directivas en instituciones educativas de educación primaria de Pisco. Recuperado de http://investigacion.pucp.edu.pe/mesinvestigacion/investigacion/competenciasdirectivas-en-instituciones-educativas-deeducacion-primaria-de-pisco

[34] Yukl, G. (2008). Leadership in Organizations. Upper Saddle River, Nueva Jersey, Estados Unidos: Prentice Hall. 


\section{Management Competencies and Trust in a Private Service Company}

Luis Martín CORTEZ Giraldo ${ }^{1}$

\begin{abstract}
This non-experimental, descriptive and correlational research aims at identifying the relationship between the management competencies of middle managers and trust within the work teams of a private services company. The sample consisted of the total population of the company. The management competencies scale of Garcia-Lombardia, Cardona and Chinchilla and the trust dimension of the Lencioni team assessment scale were used; both with adequate psychometric properties for the study. The results show with a $99 \%$ probability that trust is significantly and positively related to management competencies, i.e., the greater development of management competencies, the greater the trust within the work teams; similarly, if management competencies are less developed, the lower the level of trust.
\end{abstract}

Keywords: management competencies; trust; work teams; strategic competencies; interpersonal competencies.

\section{INTRODUCTION}

There is no doubt that the effectiveness of work teams is an important aspect for the good performance of employees within an organization, and trust is one of the success factors in achieving results among the members of the company.

Trust is based on the behaviors of managers and bosses, which favors communication, teamwork, performance, satisfaction and commitment of collaborators, and consequently makes the organization more productive (Cardona \& Wilkinson, 2009; Chand, 2018).

Lencioni (2003) defines trust as the conviction that the intentions of the team members are good; therefore, there is no reason to be careful when speaking about the mistakes, defects and limitations of the team members.

If there is trust in a team, members can speak up and be aware of their shortcomings and turn them into opportunities for improvement in order to enhance the team's development and effectiveness. Similarly, where trust exists, there is the possibility of asking for help and expressing ideas or opinions about what is happening within the team and the organization. Therefore, trust is a fundamental aspect for the development of the organization; leaders are the ones who promote the proper relationships based on high levels of trust (Yukl, 2008).

Searle and Ball (2004) address the importance of trust within organizations and emphasize that it is a fragile construct within companies, in view of the complexity of the process of building it or rebuilding it if an event causes a trust breakdown in the relationship between the employee and the organization.

Architect by Universidad Ricardo Palma. Currently working as independent consultant in Organizational Behavior and Leadership. Senior coach. (Lima, Peru) ORCID: https://orcid.org/0000-0002-5176-6757 Corresponding author: luis.cortez.g@hotmail.com 
Organizational trust is built when workers channel their personal trust towards the company, which results in the creation of bonds and, and according to the dependence and interaction developed. It can be analyzed based on the trust placed in the supervisor and the trust generated about the institution; the former refers to the trust that a subordinate has to follow instructions in the face of actions that he or she fails to control, while the latter refers to the trust in the support and fairness from the company's authorities (Mollering et al., 2004; Perilla \& Gómez, 2017).

Management competencies play an important role in the development of organizations. Cardona (1999) defines them as the day-to-day, visible behaviors of a person that lead to the achievement of results in his or her managerial role, and classifies them into three groups of competencies: strategic, interpersonal, and personal effectiveness.

Strategic competencies generate economic value; interpersonal competencies enhance the capabilities of employees and strengthen their commitment to the organization; and personal effectiveness competencies help employees develop attitudes and skills that enable them to relate to each other effectively (Cardona, 1999).

As important elements in the development of any organization, trust within work teams and management competencies lead to the objective of this study: Determine the relationship that may exist between these constructs and identify the management competencies that have the greatest impact on said relationship.

Research on management competencies have been focused on determining the most important competencies for managers in different organizations (García-Lombardía, Cardona \& Chinchilla, 2001; Tafur \& Carrera, 2012), as well as the existing differences in the level of development of management competencies in public and private institutions (Encomenderos, 2017). Further research on management competencies aimed to identify their relationship with work or organizational climate (Angulo, 2016; Sotomayor, 2017), level of motivation (Figueroa, 2017), and management autonomy and leadership (Corona et al., 2017).

One study addressed the relationship between the teamwork behaviors of top and middle management and teamwork at other levels of the organization. Raes (2014) sought to identify the competencies that management teams need to develop to achieve the cohesion and performance necessary to favorably influence the motivation, functioning, and performance of middle and operational teams.

Some research studies, focusing on the study of teamwork, include trust among the dimensions that should be assessed, as is the case of the study by Miranda and Ortega (2016).

As for the trust variable, the research by Cardona and Wilkinson (2009) studies how trust bonds are generated in the manager-collaborator relationship and identifies the behaviors —of the manager and the coworker - that contribute to stimulate what the authors call the "virtuous circle of trust building".

There were no studies that simultaneously studied management competencies and trust in work teams, hence the importance and foundation of this research.

The main objective of this research is to identify the relationship between trust and management competencies; its specific objectives are to identify the relationship between trust and strategic competencies, interpersonal competencies and personal effectiveness competencies.

The general hypothesis is as follows:

- There is a significant and positive relationship between trust and management competencies.

The specific hypotheses are as follows:

- There is a significant and positive relationship between trust and strategic competencies.

- There is a significant and positive relationship between trust and interpersonal competencies.

- There is a significant and positive relationship between trust and personal effectiveness competencies.

This research is important as it provides information on the relationship between trust and management competencies, which in turn provides a better understanding of these aspects in the organizational context and, on this basis, can be used to formulate new strategies to improve the conditions of one or both of these variables within a company or institution. As for scientific knowledge, this study can be used as a baseline for future research on the variables studied. Needless to say, the study should be replicated at a national level, including public and private organizations. 
In terms of scope, this research is limited to institutions with similar characteristics; therefore, it cannot be applied equally to all organizations, considering that public institutions have different circumstances.

\section{Management competencies}

Management competencies can be discussed based on the first contributions made by McClelland (1973), who stated that success within an organization not only depends on aspects such as personality or the aptitudes shown by the subjects, but also on a set of competencies or skills that people have for specific tasks, which are used to achieve the objectives set. This dimension of competencies allows the subject to efficiently and effectively perform a given action (Pereda et al., 2002; Cáceres \& Gallegos, 2018).

According to Hellriegel et al. (2010), managerial or management competencies are a group of competencies that involve the knowledge, skills, attitudes and behaviors required by an executive to achieve success within an organization. Properly managed, these factors, along with an adequate development of intellectual capacity, will lead to effective management and the desired performance.

Some authors describe these competencies as management skills. Riera (2005) explains that managerial skills can be learned by any individual interested and able to do so, and are based on how the individual relates to his or her environment and how he or she effectively carries out his or her activities. These skills are focused on communication, motivation, critical analysis, stress management, leadership and how individuals manage their knowledge to achieve the objectives set within the organization.

Several authors have classified management competencies, however, this research is based on the work of Cardona (1999), who includes among management competencies strategic competencies, oriented to the generation of economic value; interpersonal competencies, which enable the professional development of collaborators and the strengthening of commitment and trust in the organization; and personal effectiveness competencies, which contribute favorably to interpersonal relationships.

Strategic competencies include business vision, problem solving, resource management, customer orientation, effective relationship networking and negotiation; interpersonal competencies include communication, organization, empathy, delegation, coaching and teamwork; and personal effectiveness competencies include proactivity, self-governance, personal management and personal development (Cardona, 1999).

\section{Trust}

The term "trust", from an organizational perspective, has a variety of definitions according to the studies that have been conducted following different approaches, among which Gilbert (1998, as cited in Salinas, 2012), who defines trust as a belief held by a person within the organization, where such belief is recognized and honored, so that the subject has a high commitment to the organization, stands out. In addition, Maguire and Phillips (2008) include the term "expectation" within the definition of trust, understanding it as a factor that generates expectation in the subject within the institution and, as a result, develops predictable behaviors and a sense of goodwill towards their work center, thus obtaining better results (Rodriguez, 2018; Olvera et al., 2017).

Albrecht and Wackernagel (as cited in Santiago, 2001) define trust as the way in which an individual views other individuals within an organization, which is a dependent and consistent behavior for the structure of that person's judgments and the way he or she acts. In the opinion of Lara-Rosano (2011), trust consists of a set of formal and informal mechanisms that are developed as a result of the interaction between people in an organization through various hierarchical means, and influences the organization's strategic processes. Trust has certain characteristics that provoke values in people and generate new ways of relating, working and organizing themselves, which largely defines organizational behavior within a company.

On this basis, it can be considered that trust is of utmost importance in any organization, since it involves a series of processes that determine success within the organization. Furthermore, it can be stated that trust is a vital social capital for any organization as it is the product of a continuous and dynamic process of interpersonal relationships within organizations, which develops when individuals manage to transfer their personal trust to the organization itself (Mollering et al., 2004). Such bonds will foster a pleasant environment during the development of activities, so that any activity undertaken may contribute to a subject achieving an adequate level of confidence; thus, a person within a company can develop in a better way, enhancing his or her strengths and promoting conditions to achieve the company's objectives more effectively.

By contrast, Castaingts (2008) mentions that each of the workers within the organization requires a 
guide that orients their feelings and emotions when performing any action; these people are generally guided by beliefs that depend on the perspective of the person regarding how one relates to and understands the world.

Another important aspect regarding trust is that which defines it as the primary source of cooperation; however, it may not always be given in the best way, since a person does not necessarily receive what he or she gives to others; therefore, granting trust to a person does not necessarily mean that he or she will respond in the same or in a good way. This is a very frequent situation and is related to the personality of each individual (Acedo \& Gomila, 2013). In this regard, Alter (2002) points out the existence of selfish behavior among coworkers, an issue that should be addressed if it occurs, as trusting relationships promote cooperation, and cooperation can provide the best results regarding the performance of workers. Addressing this is a complex process that involves a series of variables and even the personality of each member of the organization.

\section{METHODOLOGY}

This is an empirical study that identifies a measurable quantity through traditional statistical tests, which allows it to be quantitative (Alfaro, 2012). It is also a descriptive study, since it analyzes events, contexts and phenomena, specifying and describing facts and characteristics of what happens (Hernández et al., 2014). In addition, it is a correlational study, as it aims to identify whether or not there is a relationship between the existing trust in the work teams and the management competencies of the immediate bosses of the employees tested. The research has a non-experimental design, avoiding the manipulation of the variables under investigation, and therefore it does not attempt to analyze the influence of one variable on another using intentional methods (Hernández et al., 2014).

The population consisted of all the employees of a private service company located in the city of Lima, with a total of 40 male and female employees over 25 years of age. A census-type sampling technique was used for this study. Out of the total sample, 2 people did not attend the day of the application of the instruments and 8 did not give their consent, therefore, the scale for measuring management competencies and the scale for measuring trust in work teams were applied to a total of 30 employees.

This study was planned in a systematic and organized manner, resulting in the achievement of the research objectives. One of the first actions taken was to hold a meeting with the organization's managers to arrange for the collection of data. Thereafter, the application of the measurement scales was scheduled. The instruments used for the development of the study were the "Management Competencies Questionnaire" by García-Lombardía, Cardona and Chinchilla (2001) and the "Team Assessment Scale" by Lencioni (2003), from which only the items corresponding to the dimension of trust were used. The first instrument has a reliability of 0.76 (Cronbach's alpha) and validity of 0.76 (Kaiser Olkin) with a variance of $55.11 \%$, and the second has a reliability of 0.95 and validity of 0.93 with a variance of $59.33 \%$. These instruments were chosen because they were better adjusted to the population studied and because there is a lack of instruments that measure the components proposed in this research. Thus, it is important to conduct more studies on instruments based on this theory or to better adapt the existing instruments to the current reality.

Subsequently, an explanation of the objectives of the study and how to respond to the instruments was given to the participants who were informed that participation was voluntary and that the questionnaires were anonymous. Upon collecting the information, a database was assembled, taking into account the pertinent values, and the statistical analysis was performed once the data had been compiled and filtered. The first step was to analyze the levels of each of the studied variables using measures of central tendency; then, the distribution of the sample was calculated using the Kolmogorov-Smirnov (K-S) test, which determined the goodness of fit between two probability distributions, allowing the identification of the most suitable statistics to achieve the proposed objectives; and, finally, the Spearman's correlation was used to calculate the correlation coefficients, with which the relationship between the studied variables was determined.

\section{RESULTS}

Demographics collected from the evaluated collaborators $(\mathrm{N}=30)$ indicate that $35.7 \%(\mathrm{~N}=11)$ are male, while $63.3 \%(\mathrm{~N}=19)$ are female, as shown in Table 1.

Table 1. Number of Employees Tested According to Sex.

\begin{tabular}{|lcc|}
\hline & Number & Percentage \\
\hline Male & 11 & 36.7 \\
Female & 19 & 63.3 \\
\hline Total & 30 & 100.0 \\
\hline
\end{tabular}

Source: Prepared by the author. 
A mean of 11.4 with a standard deviation of 0.49 was obtained for trust, and a mean of 94.5 with a standard deviation of 3.28 was obtained for all management competencies. Personal effectiveness competencies obtained the highest score with a mean of 34.90 and a standard deviation of 1.32 ; then followed strategic competencies with a mean of 30.06 and a standard deviation of 1.82; and interpersonal competencies obtained a mean of 29.57 and a standard deviation of 1.43 (see Table 2). On the basis of the results obtained, it is observed that the scores obtained for each of the dimensions and variables studied in this research are high.

Table 2. General Statistics on Trust and Competencies.

\begin{tabular}{|lccc|}
\hline & N & Mean & SD \\
\hline Trust & 30 & 11.4 & 0.49 \\
Strategic Competencies & 30 & 30.06 & 1.82 \\
Interpersonal Competencies & 30 & 29.57 & 1.43 \\
Personal Effectiveness Competencies & 30 & 34.90 & 1.32 \\
Total of Management Competencies & 30 & 94.53 & 3.28 \\
\hline
\end{tabular}

Source: Prepared by the author.

A normality analysis of the sample distribution was performed (see Table 3), and it was observed that the values are significant with a confidence level of $99 \%(p=0.00 ; p<0.01)$, suggesting that the distribution is not normal. Consequently, an analysis with non-parametric statistics was performed. Spearman's correlation coefficient was used to analyze the correlations between trust and management competencies.

Table 3. Sample Distribution.

\begin{tabular}{|lccc|}
\hline & \multicolumn{3}{c|}{ Kolmogorov-Smirnov } \\
& Statistic & df & Sig. \\
\hline Total of Management Competencies & 0.31 & 30 & 0.00 \\
Trust & 0.39 & 30 & 0.00 \\
Strategic Competencies & 0.30 & 30 & 0.00 \\
Interpersonal Competencies & 0.19 & 30 & 0.00 \\
Personal Effectiveness Competencies & 0.30 & 30 & 0.00 \\
\hline
\end{tabular}

Source: Prepared by the author.

\section{Hypothesis Testing}

Table 4 shows the Spearman correlation between trust and the total score of management competencies and their respective dimensions, i.e., strategic, interpersonal and personal effectiveness competencies. The results yield values with significance equivalent to $p=0.00(p<0.01)$ and reach a $99 \%$ level of probability that there is a significant relationship between confidence and management competencies. Additionally, on the basis of the value obtained (rho $=0.72$ ) and the classification by Martínez and Campos (2015) of the range of correlation coefficients, the relationship is highly positive. Therefore, it can be said that the greater the development of management competencies, the greater the trust within the work teams, while the opposite is also true: the lower the development of the first variable, the lower the values of the second variable. As for the management competencies dimensions, a $95 \%$ level of probability $(p=0.03 ; p<0.05)$ indicates that there is a significant relationship between trust and strategic competencies, and based on the value obtained (rho $=0.40$ ), the relationship is moderately positive (Martínez \& Campos, 2015). Hence, it can be stated that the relationship between trust and competencies aimed at generating results or economic value (strategic) is similar to the relationship between trust and all management competencies; however, their relationship is not as strong as the one cited above.

Table 4. Correlation Between Trust and Management Competencies.

\begin{tabular}{|llc|}
\hline & \multicolumn{2}{c|}{ Trust } \\
& rho & p \\
\hline Strategic Competencies & $0.40^{*}$ & 0.03 \\
Interpersonal Competencies & $0.72^{* *}$ & 0.00 \\
Personal Effectiveness Competencies & $0.59^{* *}$ & 0.00 \\
Total of Management Competencies & $0.72^{* *}$ & 0.00 \\
\hline${ }^{*}$ Correlation is significant at the level 0.05 & (2-tailed). \\
** Correlation is significant at the level 0.01 (2-tailed). \\
Source: Prepared by the author.
\end{tabular}

A 99\% level of probability ( $p=0.00 ; p<0.01$ ) shows that there is a significant relationship between confidence and interpersonal competencies, and confidence and personal efficacy competencies; however, a difference in the interpretation of these significant relationships exists because of the rho values obtained. Regarding interpersonal competencies, a rho $=0.72$ was found, thus it can be stated that there is a high positive relationship between trust and these competencies related to knowing how to develop the competencies and skills of employees (Martínez \& Campos, 2015). Regarding personal effectiveness competencies, the value found is rho $=0.59$, so it can be said that there is a moderate positive relationship between trust and these competencies that allow middle managers to adequately manage interpersonal relationships and encourage their teams to be receptive and identify with the organization (Martínez \& Campos, 2015). 


\section{DISCUSSION}

Research results show that there is a significant and positive relationship between management competencies and trust within work teams, i.e., the higher the development of management competencies, the higher the trust within work teams; and conversely, the lower the development of management competencies, the lower the level of trust among work team members.

As demonstrated by Angulo (2016) and Sotomayor (2017), the level of development of the management competencies of team leaders in an organization is directly and positively related to work climate. Their research focuses on the development of communication, teamwork, leadership, etc. One of these studies recommends that managers build trusting relationships with other members of the organization to foster a better work climate (Angulo, 2016). Meanwhile, the other study recommends that managers demonstrate ethical and transparent behavior to foster a climate of trust within the organization (Sotomayor, 2017).

The development of management competencies leads to changes and/or improvements in the personal dispositions of the members of the organization; an example of this is the increase in employees' motivation levels, as shown by the results of Figueroa's study (2017). Another example is the development of competencies of top management members to improve work among peers, as a united management team; these behaviors are transferred to middle management and the rest of the organization, thereby influencing the level of trust, improving interpersonal relationships, collaborative attitude and joint performance, as shown in the study by Raes (2014).

Managers value certain management competencies over others. According to the results of the studies by Tafur and Carrera (2012) and García-Lombardía, Cardona and Chinchilla (2001), communication, teamwork and leadership, in addition to customer orientation, integrity or ethical commitment, and coaching, among others, are the competencies regarded as more valuable. The result of the latter research was that companies want their managers to have similar levels of development of the three types of management competencies - strategic, interpersonal and personal effectiveness - with an emphasis on interpersonal competencies. Such a result may complement, in terms of knowledge, the result of this study, where a high and positive relationship between interpersonal competencies and trust within work teams is observed.
Similarly, in their study for the development of a model of management competencies for management and leadership autonomy in supervisors, Corona et al. (2017) found that interpersonal competencies were the most valued competencies. Such research can also contribute to the results obtained in this research.

On the other hand, trust together with productive conflict are the dimensions of teamwork that influence organizational change processes the most, as shown in the study by Miranda and Ortega (2016).

Likewise, the bond of trust between manager and employee has a dynamic that is based on the behaviors of those who lead the teams in the organizations; if these behaviors generate trust in the employee, the latter "tiende a corresponder con conductas también positivas" [tends to behave positively as well], as indicated in the study by Cardona and Wilkinson (p. 23, 2009). In organizational behavior, employee behavior can be predicted from observing the behavior of the leaders.

From the analysis, it can be said that, if there is greater trust in a work environment within a work team, it is likely that the direct manager of that team has a higher level of development of management competencies; thus, according to this research, interpersonal competencies are the most important, followed by strategic competencies and personal effectiveness competencies. As this relationship is direct and positive, it can be deduced that, within an organization in which a manager lacks management competencies, it is likely that there is little trust among the members of the work team under his or her responsibility. It should be noted that the achievement of higher levels in one of the variables is not based solely on the development of the other variable; although a relationship between trust and management competencies has been found, it is not possible to state a single cause for any of them.

If an organization needs to improve the performance of its employees, it should focus on developing the management competencies of the people in charge of them and thus increase the level of trust within their teams to improve employees' performance and increase their ability to achieve the company's objectives.

\section{CONCLUSIONS}

1. A significant and positive relationship exists between trust and management competencies, thus it can be concluded that the higher 
the levels of management competencies, the higher the levels of trust among employees.

2. A significant and positive relationship exists between trust and strategic competencies, thus it can be concluded that the higher the levels of strategic competencies, the higher the levels of trust within the work teams.

3. A significant and positive relationship exists between trust and interpersonal competencies, thus it can be concluded that the higher the levels of interpersonal competencies, the higher the levels of trust within the work teams.

4. A significant and positive relationship exists between trust and personal efficacy competencies, thus it can be determined that the higher the levels of personal effectiveness competencies, the higher the levels of trust among the members of the work teams.

5. The results can be applied to organizations with similar characteristics to the company where this study was conducted.

6. These findings are relevant since, in order to increase workers' trust, the management competencies of their leaders will have to be strengthened, thereby leading to a series of benefits in the development of the organization

\section{REFERENCES}

[1] Acedo, C., \& Gomila, A. (2013). Confianza y cooperación. Una perspectiva evolutiva. Contrastes, 18, 221-238.

[2] Alfaro, C. (2012). Metodología de investigación científica aplicado a la ingeniería [Final research project report]. Instituto de Investigación de la Facultad de Ingenierías Eléctrica y Electrónica de la Universidad Nacional del Callao. Retrieved from https://unac.edu.pe/ documentos/organizacion/vri/cdcitra/Informes Finales_Investigacion/IF_ABRIL_2012/IF_ ALFARO\%20RODRIGUEZ_FIEE.pdf

[3] Alter, N. (2002). Théorie du don et sociologie du monde du travail. Revue du MAUSS, 2(20), 263-285. Retrieved from https://doi. org/10.3917/rdm.020.0263

[4] Angulo, D. (2016). Determinación de las competencias directivas y del clima laboral existente en el I.E. "Los Positos", Mórrope, Lambayeque. (Undergraduate thesis). Universidad Católica Santo Toribio de
Mogrovejo, Chiclayo. Retrieved from http:// tesis.usat.edu.pe/handle/20.500.12423/164

[5] Cáceres, L., \& Gallegos, W. (2018). Importancia de las competencias directivas desde la percepción de los empresarios arequipeños. Contabilidad y Negocios 13(26), 109-128. Retrieved from https://doi.org/10.18800/ contabilidad.201802.007

[6] Cardona, P. (December, 1999). En busca de las competencias directivas. IESE Revista de antiguos alumnos, 19-26.

[7] Cardona, P., \& Wilkinson, H. (2009). Cómo crear el círculo virtuoso de la confianza. IESE Insight, (3), cuarto trimestre 20-27.

[8] Castaingts, J. (2008). Antropología simbólica y neurociencia. Alteridades, 18(35), 129-138.

[9] Chand, S. R. (2018). Inspira: Cómo crear una cultura organizacional poderosa. Pensilvania, Estados Unidos de América: Whitaker House.

[10] Corona, L., Ramírez, G., \& Vázquez, E. (2017). Modelo de competencias directivas para la autonomía de gestión y liderazgo en supervisores de educación básica en el marco de las reformas educativas en México. Killkana Sociales, 1(3), 23-28. Retrieved from https:// doi.org/10.26871/killkana_social.v1i3.59

[11] Encomenderos, Y. (2017). Competencias directivas en instituciones educativas públicas y privadas del distrito de Tarapoto, 2017. (Master thesis). Universidad César Vallejo, Lima. Retrieved from http://repositorio.ucv.edu. pe/handle/UCV/12785

[12] Figueroa, J. (2017). Competencias directivas y nivel de motivación en docentes de secundaria de instituciones educativas privadas, Bellavista - Callao, 2015. (Master thesis). Universidad César Vallejo, Lima. Retrieved from https://repositorio.ucv.edu.pe/ handle/20.500.12692/8344

[13] García-Lombardía, P., Cardona, P., \& Chinchilla, M. (2001). Las competencias directivas más valoradas. Retrieved from https://media.iese. edu/research/pdfs/OP-01-04.pdf

[14] Hellriegel, D., Jackson E., \& Slocum J. (2010). Administración: Un enfoque basado en competencias. Ciudad de México, Mexico: Cengage Learning Editores.

[15] Hernández, R., Fernández, C., \& Baptista, P. (2014). Metodología de la Investigación (6 $6^{\text {th }}$ ed.). México D.F., Mexico: McGraw-Hill. 
[16] Lara-Rosano, F. (2011). Organizaciones Complejas. En J. Flores y G. Martinez (Compiladores), Encuentros con la complejidad. Ciudad de México, Mexico: Siglo XXI Editores.

[17] Lencioni, P. (2003). Las cinco disfunciones de un equipo. Barcelona, Spain: Ediciones Urano.

[18] Maguire, S., \& Phillips, N. (2008). Citibankers at Citigroup: a study of the loss of institutional trust after a merger. Journal of Management Studies, 45(2), 372-401. Retrieved from https:// doi.org/10.1111/j.1467-6486.2007.00760.x

[19] Martínez, A., \& Campos, W. (2015). Correlación entre actividades de interacción social registradas con nuevas tecnologías y el grado de aislamiento social en los adultos mayores. Revista mexicana de Ingeniería Biomédica, 36(3), 181-190.

[20] McClelland, D. (1973). Testing for Competence rather than for "Intelligence". American Psychologist, 28(1), 1-14.

[21] Miranda, A., \& Ortega, M. (2016). Evaluación de las características de trabajo en equipo en las empresas del sector educativo particular de la ciudad de Cajamarca-2016. (Undergraduate thesis). Universidad Privada Antonio Guillermo Urrelo, Cajamarca. Retrieved from http:// repositorio.upagu.edu.pe/bitstream/handle/ UPAGU/258/IT\%20Alexis\%20y\%20Miguel. pdf? sequence=1\&isAllowed=y

[22] Mollering, G., Bachmann, R., \& Lee, S. H. (2004). Understanding organizational trust - foundations, constellations, and issues of operationalisation. Journal of Managerial Psychology, 19(6), 555-570. Retrieved from http://dx.doi.org/10.1108/02683940410551480

[23] Olvera, J., LLorens, S., Acosta, H., \& Salanova, M. (2017). El liderazgo transformacional y la confianza como antecedentes del desempeño en equipo en el ámbito sanitario. Anales de Psicología/Annals of Psychology, 33(2), 365375.

[24] Pereda, S., Berrocal, F., \& López, M. (2002). Gestión de recursos humanos por competencias y gestión del conocimiento, Dirección y Organización, (28), 43-54.

[25] Perilla, L., \& Gómez, V. (2017). Relación del estilo de liderazgo transformacional con la salud y el bienestar del empleado: el rol mediador de la confianza en el líder. Revista de Psicología del Trabajo y de las Organizaciones, 33(2), 95108.
[26] Raes, A. (2014). Predicar con el ejemplo. El trabajo en equipo empieza en la alta dirección. Revista IESE Insight, (20), 31-38.

[27] Riera, J. (2005). Habilidades en el deporte. Barcelona, Spain: INDE Publicaciones.

[28] Rodríguez Egea, N. (2018). Liderazgo auténtico, clave de Confianza. (Master's final paper). Universitat Jaume I, Castelló de la Plana. Retrieved from http://repositori.uji. es/xmlui/bitstream/handle/10234/180476/ TFM_2018_RodriguezEgea_Natalia. pdf? sequence=1\&isAllowed=y

[29] Salinas, M. (2012). Elementos que influyen en el establecimiento de la confianza dentro de las organizaciones. (Doctoral thesis). Universidad Autónoma de Nuevo León, San Nicolás de Garza.

[30] Santiago, A. (2001). Desarrollo y validación de la escala de confianza organizacional. (Doctoral thesis). Pontificia Universidad Católica de Puerto Rico, Ponce.

[31] Searle, R., \& Ball, K. (2004). The Development of Trust and Distrust in a Merger. Journal of Managerial Psychology, 19(7), pp. 708-721. Retrieved from https://doi. org/10.1108/02683940410559392

[32] Sotomayor, L. (2017). Competencias directivas y clima organizacional en instituciones educativas del distrito El Agustino, 2015 (Master thesis). Universidad César Vallejo, Lima. Retrieved from http://repositorio.ucv.edu. pe/handle/UCV/8323

[33] Tafur, R., \& Carrera, P. (2013). Competencias Directivas en instituciones educativas de educación primaria de Pisco. Retrieved from http://investigacion.pucp.edu.pe/mesinvestigacion/investigacion/competenciasdirectivas-en-instituciones-educativas-deeducacion-primaria-de-pisco

[34] Yukl, G. (2008). Leadership in Organizations. Upper Saddle River, New Jersey, United States: Prentice Hall. 
